\title{
Buoyancy-forced circulations in shallow marginal seas
}

\author{
by Michael A. Spall ${ }^{1}$
}

\begin{abstract}
The properties of water mass transformation and the thermohaline circulation in shallow marginal seas with topography and subject to surface cooling are discussed in the context of an eddy-resolving primitive equation model and an analytic planetary geostrophic model. A unique and important aspect of the model configuration is that the geostrophic contours, or characteristics of the system, extend from a region where temperature is restored toward a uniform value, providing a source of heat, through the cooling region. This removes a degree of symmetry that has often been imposed in previous studies of deep convection. The heat loss within the marginal sea is balanced by lateral advection from the restoring region. The planetary geostrophic model shows that the basic temperature distribution can be well predicted by integrating along geostrophic contours from their entry into the marginal sea to their exit. Scaling estimates for the exchange rate and density of the waters formed within the marginal sea are derived and compare well with a series of numerical model calculations. In contrast to many previous buoyancy-forced deep convection problems, basin-scale cooling is balanced mainly by the mean flow, with mesoscale eddies serving primarily to restratify locally but not to provide a net heat flux to balance cooling. However, eddy fluxes and the mean flow are locally of comparable importance for cases with a localized patch of surface cooling.
\end{abstract}

\section{Introduction}

Marginal seas are often regions of important water mass transformations. Although small in area, the net buoyancy flux due to heat loss or evaporation in marginal seas can produce large volumes of dense waters with distinct signals in temperature, salinity, and potential vorticity that can be traced far from their source. These water masses transport significant amounts of heat and salt, and thus play an important role in the global thermohaline circulation.

There has been much recent interest in the dynamics and thermodynamics of water mass transformation and deep convection; a recent review is given by Marshall and Schott (1999). It has become apparent that lateral heat transport by mesoscale eddies often plays a crucial role in balancing surface cooling (e.g. Visbeck et al., 1996; Chapman and Gawarkiewicz, 1997; Pringle, 2001; Spall, 2004). The relationship between the heat carried by the eddies and their generation by baroclinic instability allows one to derive estimates for various properties of the water mass transformation, such as the density of the

1. Department of Physical Oceanography, Woods Hole Oceanographic Institution, Woods Hole, Massachusetts, 02543, U.S.A.email: mspall@whoi.edu 
waters formed, the depth of convection, and the restratification time (Marshall and Schott, 1999).

One common aspect of these studies is that the model configuration has had a degree of symmetry in the along mean flow direction. For the cases of localized cooling (Visbeck et al., 1996; Marshall and Schott, 1999) the mean rim-current that arises around the region of cooling is two dimensional. Because the mean streamlines are closed, such symmetry prohibits the generation of a mean flow through the cooling region, leaving eddy fluxes as the only viable mechanism to balance the surface cooling. Other studies have used a periodic channel (Pringle, 2001), or a closed basin (Condie and Rhines, 1994) which also imposes some degree of symmetry along the mean flow direction and makes it difficult for the mean flow to advect heat. Such a symmetry may be broken by introducing regions of heating and cooling along closed streamlines (e.g. Nilsson et al., 2005, this issue) in which case a steady barotropic flow along closed topographic contours can balance surface fluxes and mixing.

If the ocean is shallow enough, or the cooling strong enough, deep convection can reach all the way to the bottom. Examples of such shallow marginal seas include the northern Adriatic Sea, the Mediterranean Sea, and regions of the Arctic and Antarctic shelf. In such cases, the sloping bottom plays an important role in the advection of dense waters away from their formation region (e.g. Rhines, 1998). In addition, information often propagates along geostrophic contours, which are strongly controlled by the bottom topography (Straub and Rhines, 1990). The bottom slope may not only play an important role in the transport of dense water away from the deep convection site, but it may also provide a pathway for source waters to be advected into the region of deep convection. Thus, the addition of bottom topography may provide a mechanism to compete with eddy fluxes to balance surface cooling.

The purpose of the present study is to explore the influences of a bottom slope and a restoring region on the water mass transformation process in a shallow marginal sea. Shallow in this context indicates that convection reaches the bottom. A key aspect of the model configuration is that the entire cooling region is connected to the restoring region by geostrophic contours.

\section{Buoyancy-forced circulation in an idealized marginal sea}

The thermohaline circulation in an idealized marginal sea will first be explored using the MIT primitive equation numerical model (Marshall et al., 1997). The model solves the momentum and density equations using level (depth) coordinates in the vertical and a staggered $\mathrm{C}$-grid in the horizontal. The model retains a free surface. A linear equation of state is used so that density is proportional to temperature as $\rho=\rho_{0}-\alpha T$, where $\rho_{0}$ is a reference density for seawater and the thermal expansion coefficient $\alpha=0.2 \mathrm{~kg} \mathrm{~m}^{-3} \mathrm{C}^{-1}$. The model is initialized at rest with a uniform temperature of $5^{\circ} \mathrm{C}$. The model is configured with horizontal grid spacing of $3 \mathrm{~km}$ and 10 levels of uniform thickness in the vertical (50 $\mathrm{m}$ for the standard case). The Coriolis parameter is $10^{-4} \mathrm{~s}^{-1}$ and constant (for orientation, east refers to increasing $x$ direction and north refers to increasing $y$ direction). 


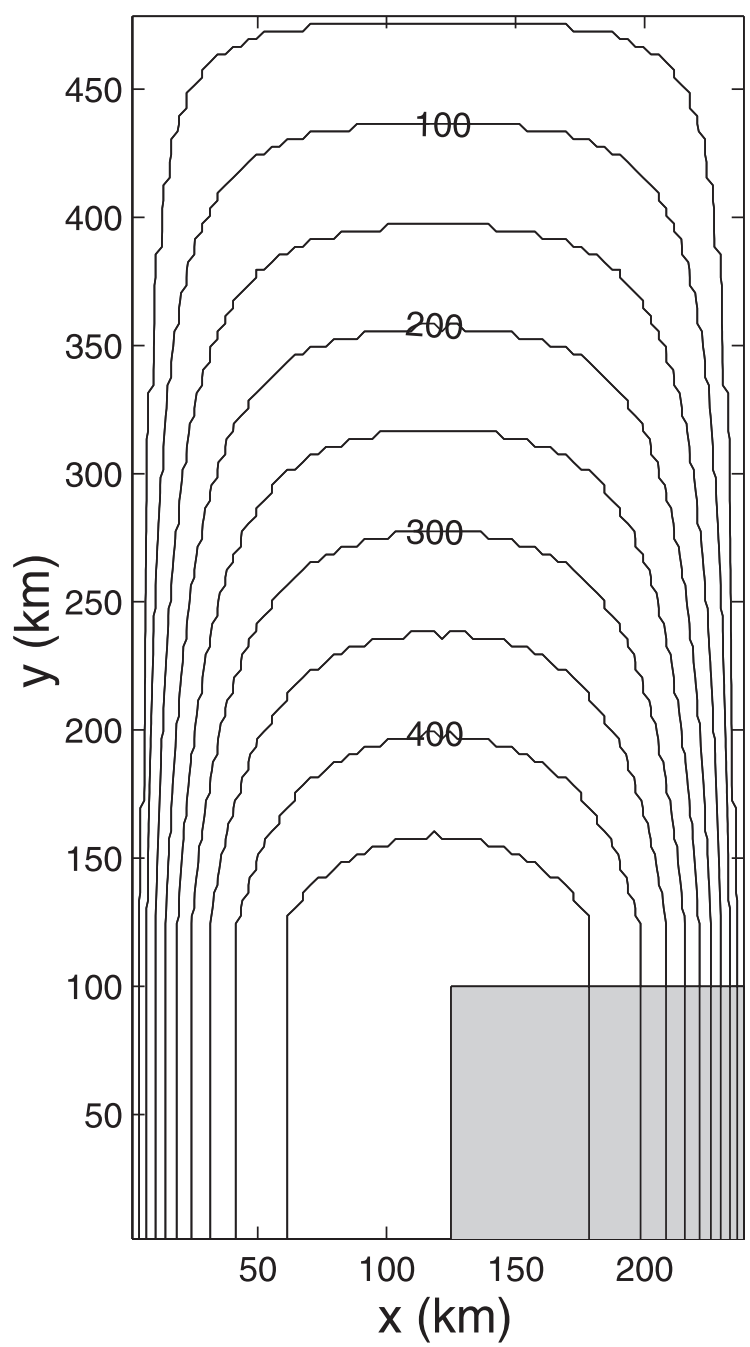

Figure 1. Basin configuration and bottom topography (contour interval $50 \mathrm{~m}$ ). The shaded region in lower right is where the model temperature is restored towards $5^{\circ} \mathrm{C}$ with time scale 100 days.

The internal deformation radius for a typical model calculation is approximately $10 \mathrm{~km}$ and reasonably well resolved by the horizontal grid. The calculations in Table 1 have also been carried out at $5 \mathrm{~km}$ horizontal resolution with 10 levels, and the central calculation has been carried out with $5 \mathrm{~km}$ horizontal resolution and 20 levels and the results are very similar to those discussed here.

The model domain for the central calculation consists of a closed rectangular basin $480 \mathrm{~km}$ long and $240 \mathrm{~km}$ wide (Fig. 1). The bottom slopes downward from $50 \mathrm{~m}$ depth on the outer boundary to a maximum of $500 \mathrm{~m}$ depth in the interior. The horizontal e-folding scale of the topography in the zonal direction $l=25 \mathrm{~km}$ and the topography varies linearly 
in the meridional direction. The temperature within the shaded region is restored toward $5^{\circ} \mathrm{C}$ throughout the water column with a time scale of 100 days. This provides a supply of warm water to balance the cooling within the marginal sea. The exchange rate with this restoring region is not specified, but instead emerges as part of the solution. The model is forced by an annual cycle of cooling of strength $120 \mathrm{~W} \mathrm{~m}^{-2}$ applied over the marginal sea north of $y=150 \mathrm{~km}$ for two months followed by no forcing for 10 months (annual mean heat loss is $20 \mathrm{~W} \mathrm{~m}^{-2}$ ). This annual cycle is repeated for a period of 10 years. The sensitivity of the water mass transformation and circulation to changes in the basin depth, bottom slope and width, strength of forcing, and Coriolis parameter will be explored in the following section. Calculations with depth contours that close within the domain at the southern end, instead of intersecting a vertical wall, produce very similar results to those discussed here. The results are also not sensitive to the zonal extent of the restoring region, provided that it encompasses all topographic contours.

Subgridscale processes are parameterized using Laplacian viscosity and diffusion in both the horizontal and vertical directions. Lateral boundary conditions are no-slip and there is no heat flux through the solid boundaries. The horizontal diffusivity and viscosity are $25 \mathrm{~m}^{2} \mathrm{~s}^{-1}$. The vertical diffusion and viscosity coefficients are $10^{-5} \mathrm{~m}^{2} \mathrm{~s}^{-1}$. Unstable density profiles are mixed vertically by increasing the vertical diffusion coefficient to $1000 \mathrm{~m}^{2} \mathrm{~s}^{-1}$. This simple parameterization neglects the influences of more complex processes such as tidal and shear mixing. There is a linear bottom drag with coefficient $C_{d}=10^{-6} \mathrm{~s}^{-1}$. The general results are not sensitive to small variations in these coefficients.

The model has been integrated until a statistical equilibrium is achieved, which takes several years. The mean temperature and horizontal velocity at $25 \mathrm{~m}$ depth averaged over the final 5 years of a 10 year simulation are shown in Figure 2. The entire model domain is shown in this figure, although subsequent figures will show only the region north of $y=$ $200 \mathrm{~km}$, away from the artificial restoring region. The general circulation through the marginal sea is cyclonic, with shallow water generally to the right of the flow. The inflow to the marginal sea is broadly distributed across the interior, while the outflow is concentrated in a narrow boundary current on the left side of the basin. A similarly cyclonic flow along topography in a region of cooling is discussed by Walin et al. (2004). The velocities in the interior are $O\left(1 \mathrm{~cm} \mathrm{~s}^{-1}\right)$. The sea-surface temperature decreases continuously along the direction of the flow so that the minimum temperature is approximately $1^{\circ} \mathrm{C}$ cooler than the inflowing water. This circulation and temperature distribution are broadly consistent with the circulation in the northern Adriatic Sea produced by a full general circulation model with realistic topography and forcing (e.g. Zavatarelli et al., 2002).

The temperature and velocity interpolated to a depth $50 \mathrm{~m}$ above the bottom are shown in Figure 3. The velocity is generally directed along topographic contours, inflowing on the right side of the basin and outflowing on the left side of the basin. The central portion of the domain is stratified (compare to Fig. 2), while the northern and western regions are less stratified. The deep circulation is confined to flow along topographic contours (except due 

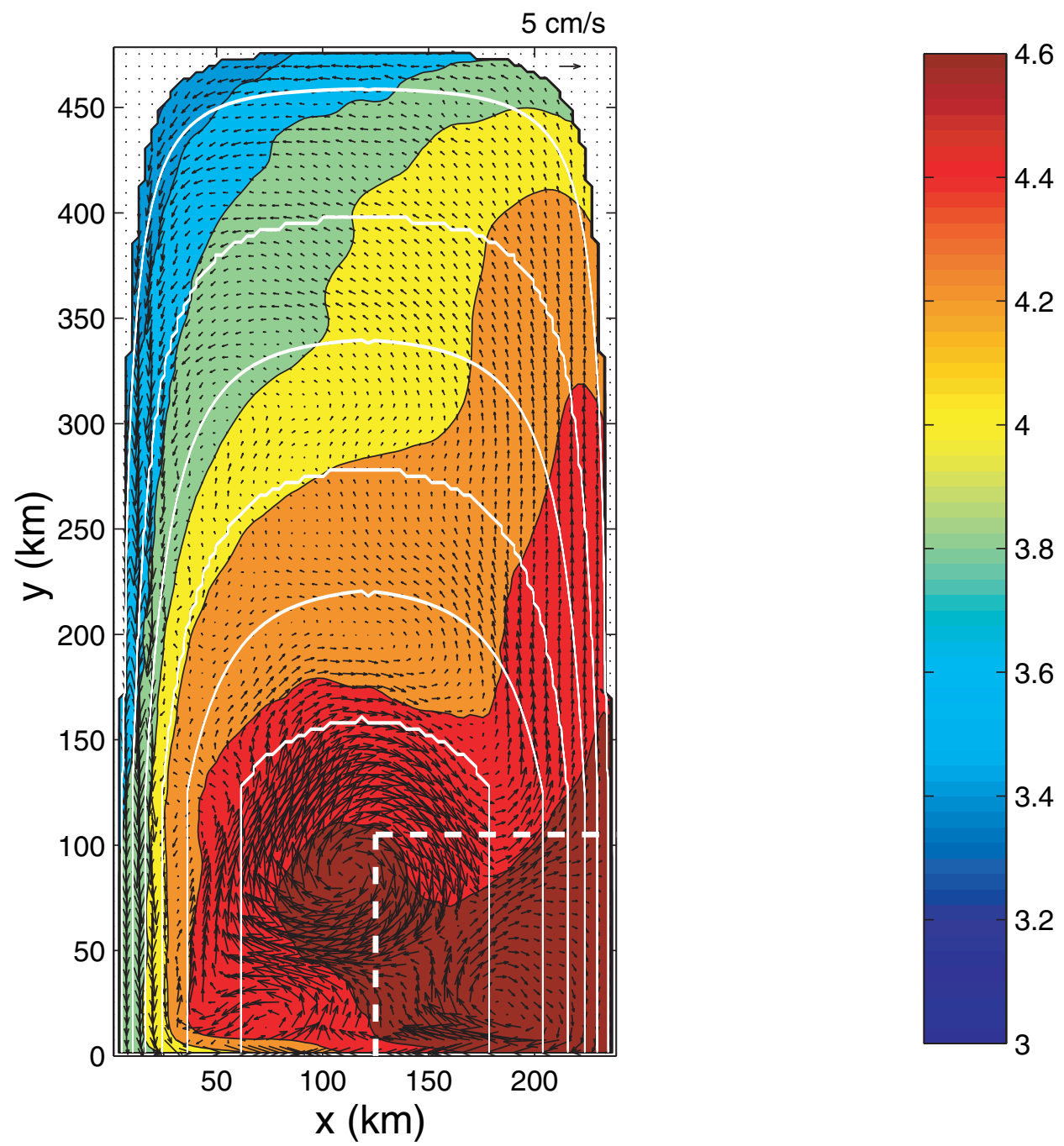

Figure 2. Mean temperature and velocity (every other grid point) at $25 \mathrm{~m}$ from the final 5 years of model integration. Bottom depth contours are indicated by solid white contours, the restoring region is indicated by the dashed white lines.

to frictional effects discussed more below), but is also required to cross temperature contours in order to balance the heat loss due to cooling. The mean velocity vector in the model rotates counter-clockwise with depth, as expected for a geostrophic flow in a region of heat loss.

There is little mean downwelling at mid-depths; essentially all of the downwelling within the basin is concentrated in the bottom Ekman layer. Mean upwelling is small everywhere within the basin (outside the restoring region). A plan view of the vertical velocity at the base of the deepest grid cell is shown in Figure 4. Even at the bottom, the 
$5 \mathrm{~cm} / \mathrm{s}$
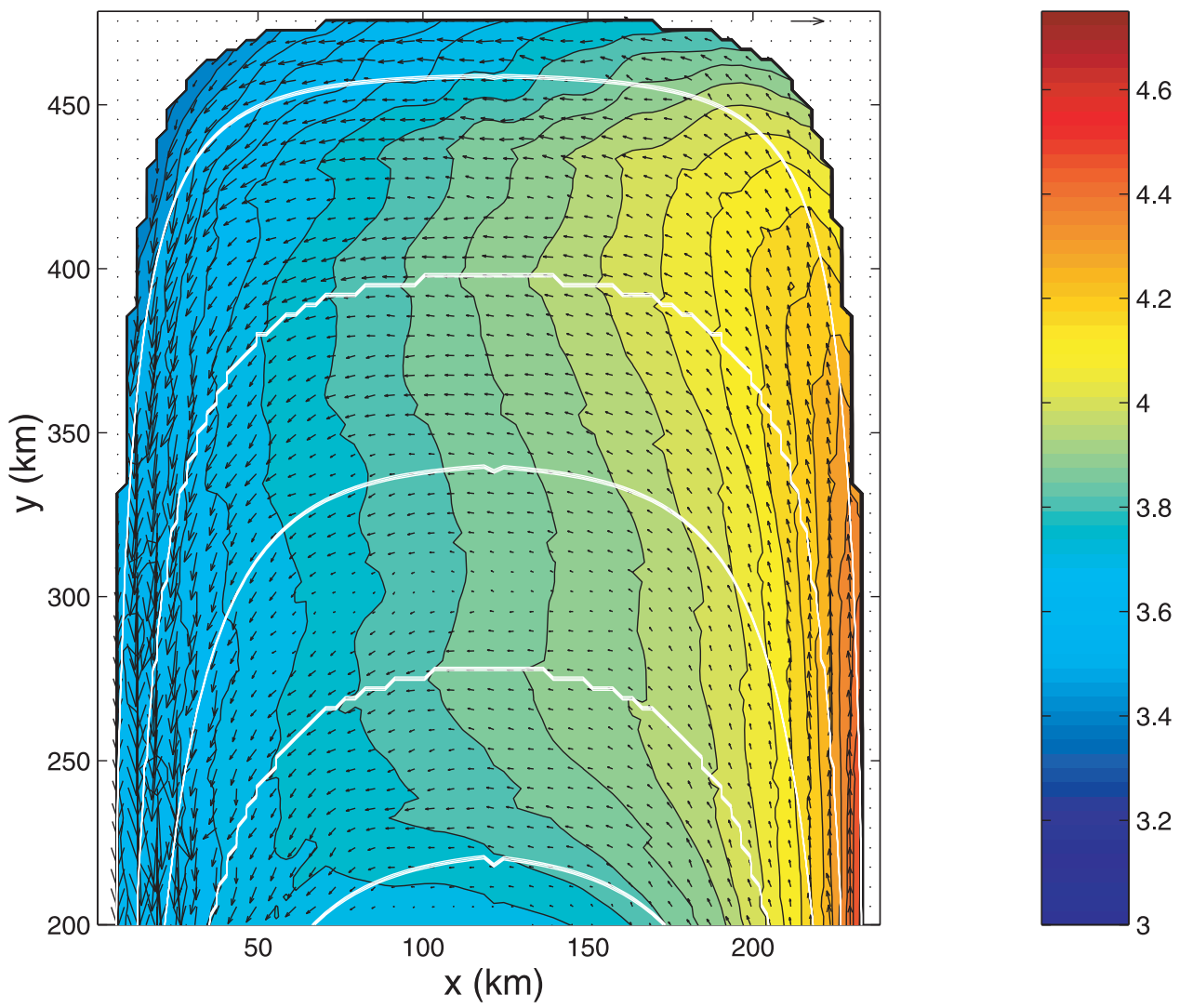

Figure 3. Mean temperature and velocity (every other grid point) interpolated to a depth $50 \mathrm{~m}$ above the bottom. Bottom depth contours are shown in white.

vertical velocity is small over most of the domain. Most of the downwelling takes place under the strong, narrow outflowing boundary current along the western side of the basin.

This circulation is much different from that found in laboratory experiments by Condie and Rhines (1994) for uniform cooling over a bowl-shaped basin. In their case, the upper-layer circulation was anticyclonic and the deep flow was cyclonic. The present flow is much more barotropic and is flowing with shallow water on the right-hand side (cyclonically). The difference is due to the source of warm water in the open ocean in the present case, which allows for a pressure gradient to develop along the direction of the flow and for a net pressure change across the opening of the marginal sea. The "open" nature of the marginal sea removes the constraint that the integral of $w$ at each depth within the marginal sea must vanish. As a result, there is no upwelling in the interior of the marginal sea, and no equivalent of the Hadley Cell described by Condie and Rhines, which forced the large-scale circulation in their laboratory experiments.

Vertical sections of temperature and the meridional velocity at $y=200 \mathrm{~km}$ are shown 


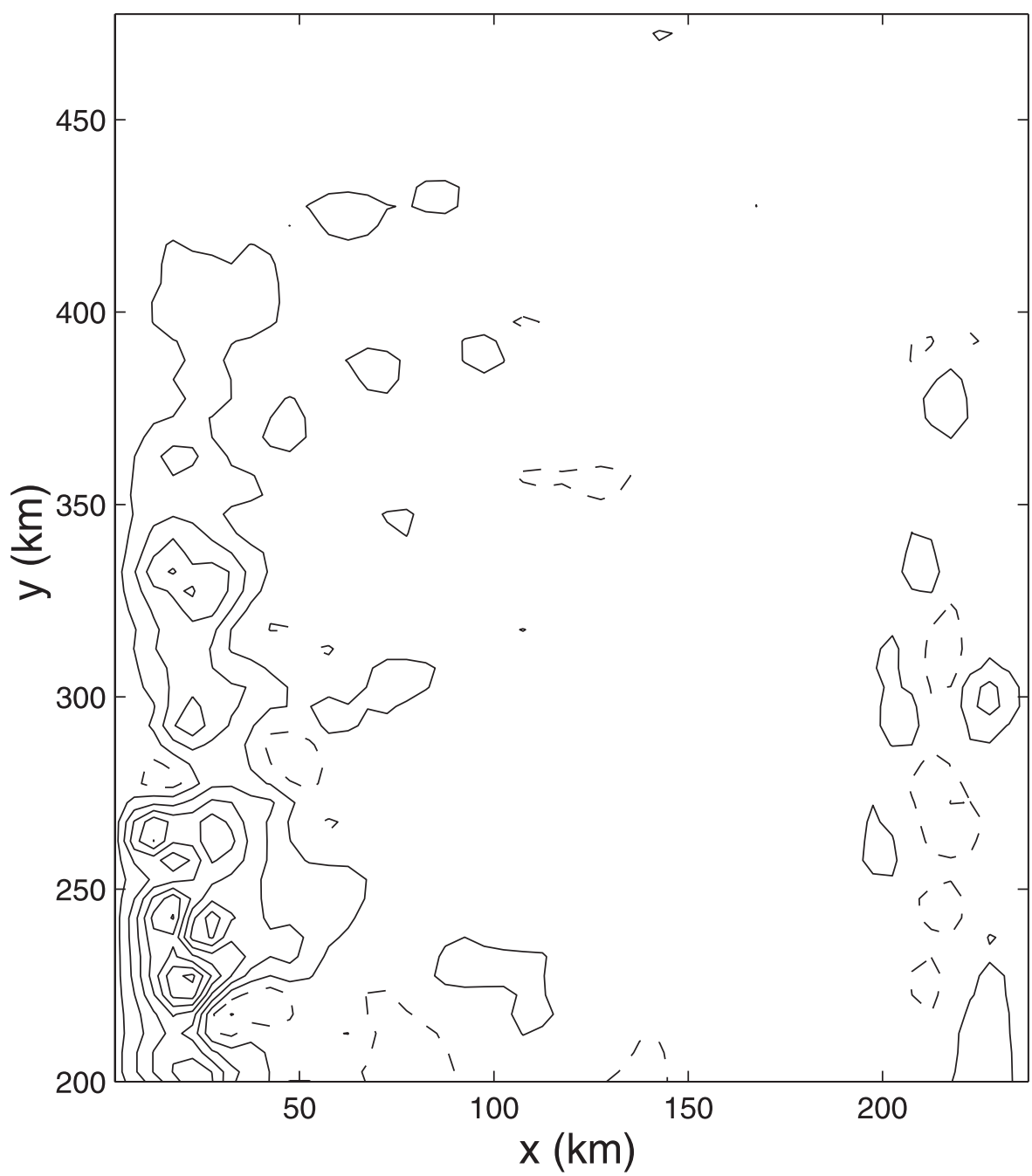

Figure 4. Mean vertical velocity at the bottom (contour interval $10^{-5} \mathrm{~m} \mathrm{~s}^{-1}$, solid contours downward).

in Figure 5. The interior and eastern portion of the marginal sea are stratified and flowing northward at all depths. The outflowing waters are much colder, more weakly stratified, and banked up against the left-hand side over the sloping bottom of the basin. The inflowing velocity is of $O\left(1 \mathrm{~cm} \mathrm{~s}^{-1}\right)$, while the outflowing velocity is an order of magnitude larger. There is a tendency for the inflowing velocity to be larger in shallower water, so that the depth integrated meridional transport is more uniform in the zonal direction than is the velocity.

Many previous studies have shown that eddies resulting from baroclinic instabilities can play a central role in balancing the heat loss to the atmosphere. Cases of localized heat loss 

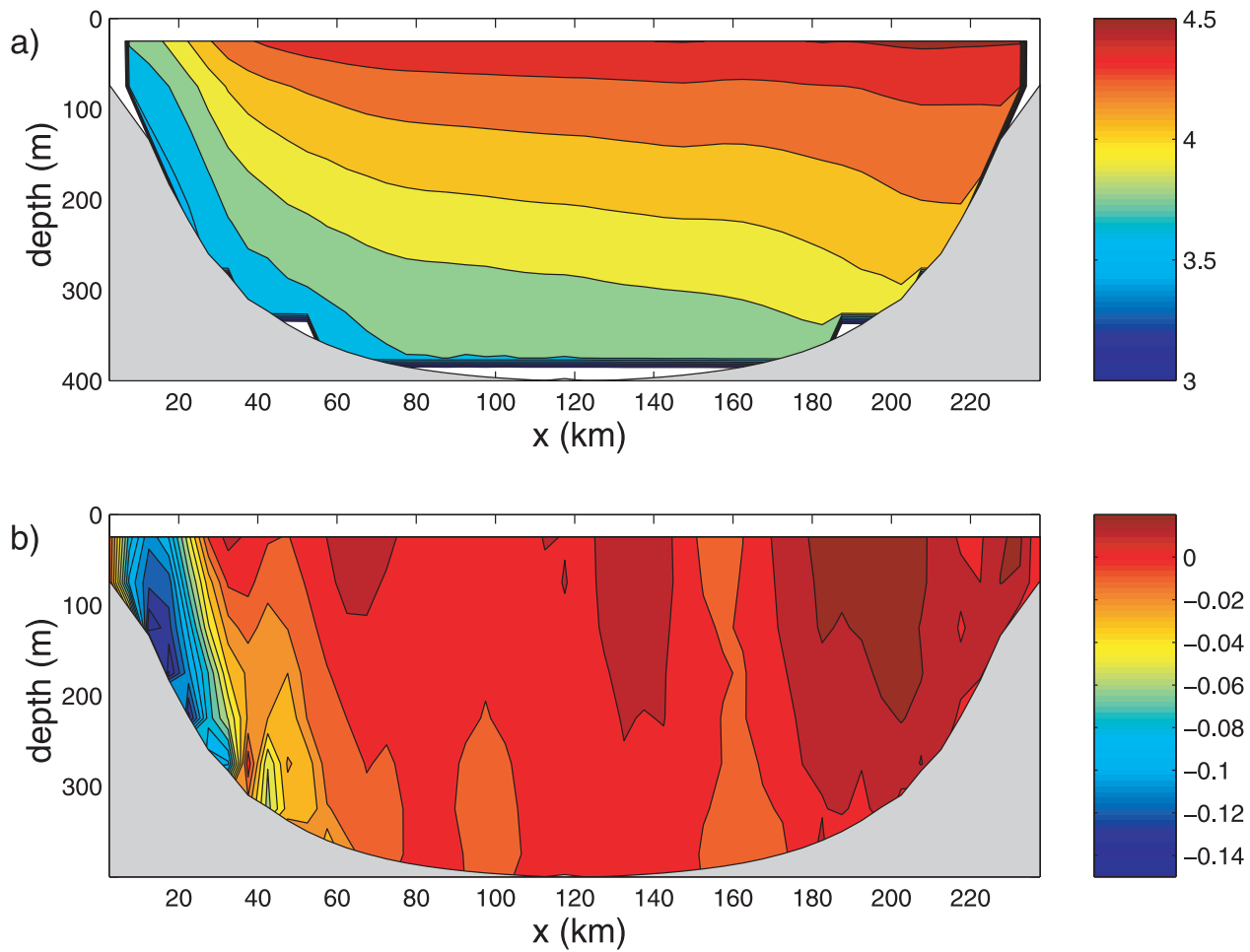

Figure 5. Mean zonal sections at $y=200 \mathrm{~km}$ for (a) temperature $\left({ }^{\circ} \mathrm{C}\right)$ and (b) meridional velocity $\left(\mathrm{m} \mathrm{s}^{-1}\right)$.

(e.g. Visbeck et al., 1996; Chapman and Gawarkiewicz, 1997) and uniform heat loss in a periodic channel (Pringle, 2001) both find that eddies are primarily responsible for balancing surface cooling. In a previous marginal sea study with topography limited to the near boundary region, Spall (2004) also found that eddies were crucial to equilibrating heat loss over the flat bottom in the interior and in determining the properties of the waters formed in the marginal sea.

Eddies are generated in late winter along the density gradients that arise in regions of deep convection. A synoptic view of the upper level temperature and velocity at the end of the cooling period is shown in Figure 6. The basin-scale temperature reflects the mean state with warm water entering from the southeast and cold water isolated in the northwest. The strong gradient regions in the north and west are characterized by mesoscale eddies with length scales of $20 \mathrm{~km}$ and velocity scales of $20 \mathrm{~cm} \mathrm{~s}^{-1}$. These time dependent eddies overwhelm the mean velocity in these regions (note the difference in velocity scale).

The relative influences of mean and eddy advection in the temperature tendency equation were calculated from the model advection terms over the final 5 years of integration. For ease of presentation, the fields have been averaged along the topographic contours in the region north of $y=200 \mathrm{~km}$ and presented as a function of depth and depth 
$25 \mathrm{~cm} / \mathrm{s}$
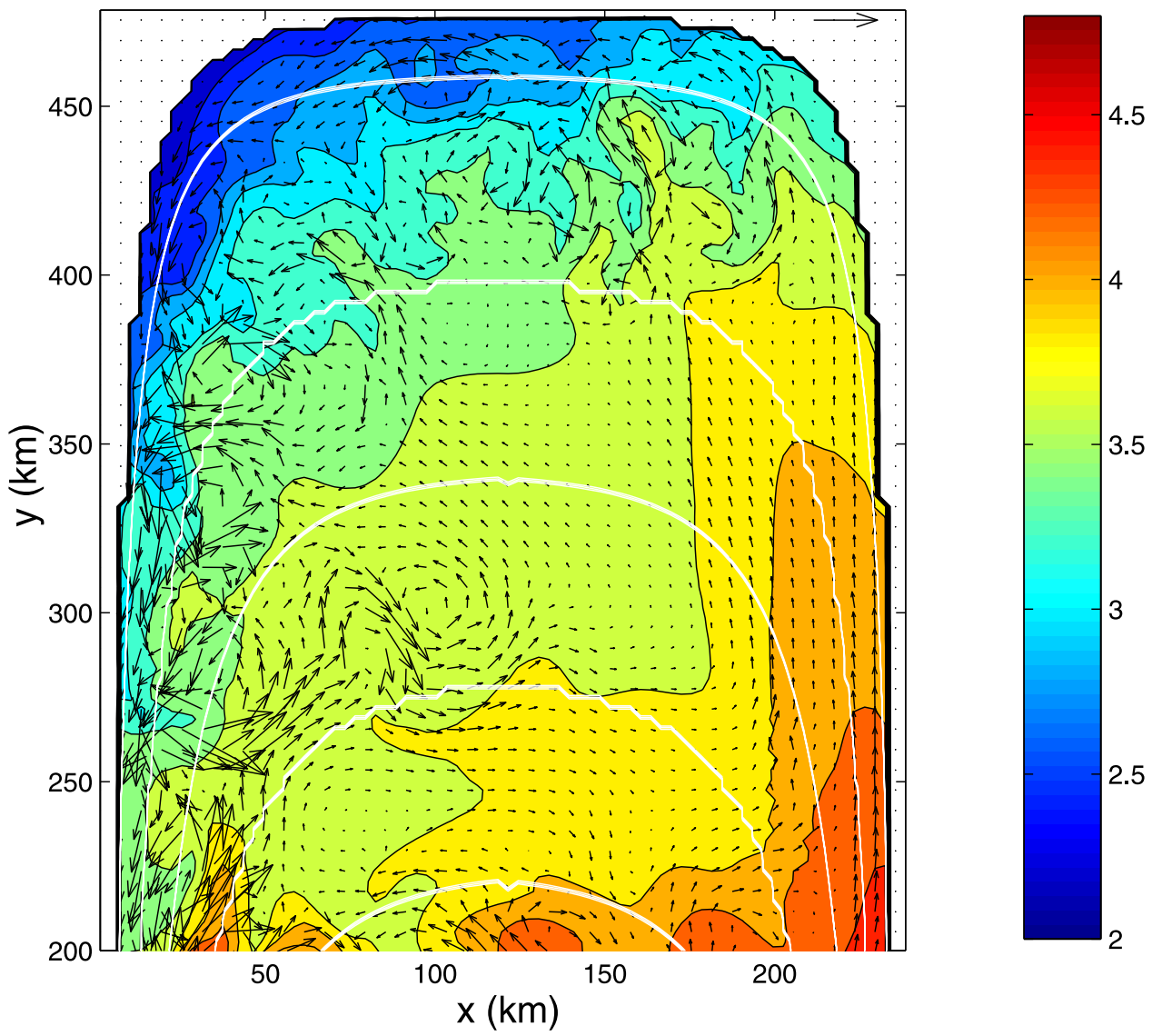

Figure 6. Temperature and velocity (every other grid point) at $25 \mathrm{~m}$ at the end of the cooling period in year 8. Bottom depth contours are shown in white.

contour (Fig. 7). (If the domain were circular, the depth contour would map directly onto the radial direction.) The sum of these terms essentially balances the local surface cooling at each point in the model domain; lateral diffusion is negligible. The mean advection is weakly depth dependent and always acts to warm the fluid. This results from the cyclonic advection of warm waters from the restoring region into the marginal sea. The magnitude is largest in shallow water because the heat loss, which is spatially uniform, is more concentrated in depth there. The eddy heat flux contribution is quite different. It is positive near the surface and negative at depth. The eddies are acting primarily to restratify the water column, not to provide a depth-integrated heat flux at a given location. A calculation with steady surface heat flux at the annual mean of $20 \mathrm{Wm}^{-2}$ and increased horizontal viscosity and diffusivity of $125 \mathrm{~m}^{2} \mathrm{~s}^{-1}$ produces a steady circulation with mean flow and sea-surface temperature that are very similar to the eddying case, yet it is everywhere 

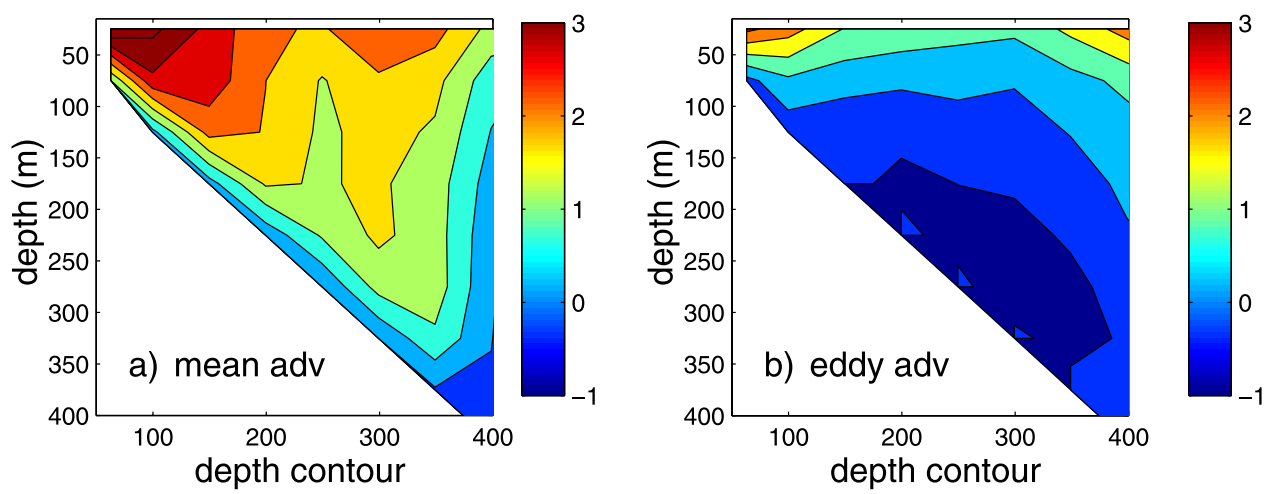

Figure 7. Contributions to the temperature tendency as a function of depth and depth contour due to:

(a) mean advection and (b) eddy fluxes (units $10^{-8}{ }^{\circ} \mathrm{C} \mathrm{s}^{-1}$ ).

unstratified. This result supports the interpretation that the primary function of the eddies is to restratify the water column, and that they do not drive the mean flow or control the basic circulation and temperature distribution.

The depth integral of the mean and eddy contributions to the temperature tendency are shown in Figure 8 . The net heat loss at the surface is balanced primarily by the mean advection, with the eddies having relatively little net contribution, except near the outer boundary and near the center of the domain ( $400 \mathrm{~m}$ isobath). The eddy fluxes over the deep topography originate at the edge of the artificial restoring region, and so are probably an

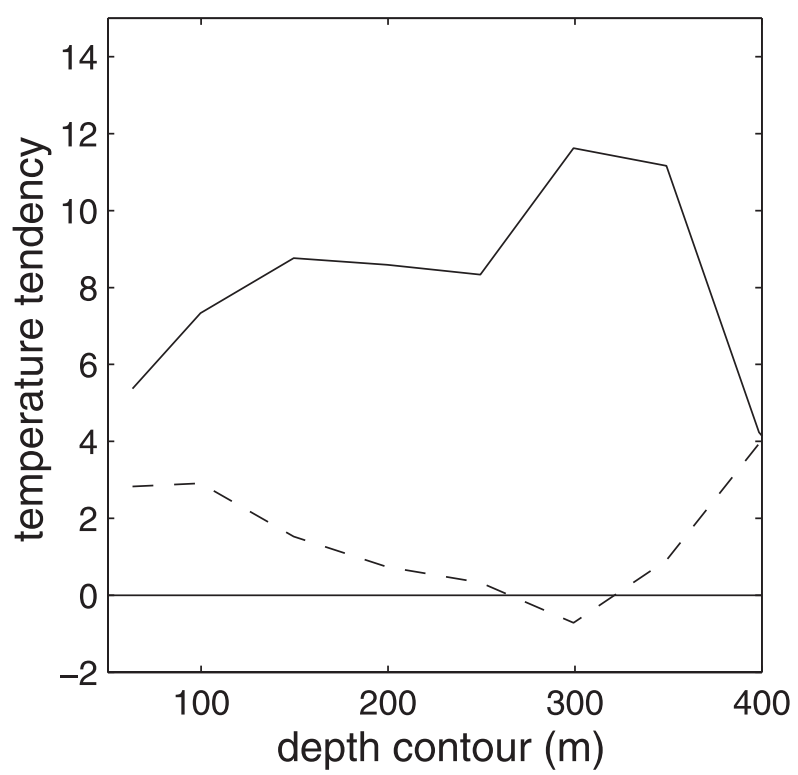

Figure 8. (a) Vertical sum of the mean (solid) and eddy (dashed) heat flux divergences as a function of depth contour north of $y=200 \mathrm{~km}$ (units $10^{-8}{ }^{\circ} \mathrm{C} \mathrm{s}^{-1}$ ). 
artifact of the model configuration. A calculation with steady forcing at the annual mean strength of the intermittent forcing used here shows even less importance for the depth-averaged eddy fluxes. This suggests that some of this eddy heat flux is due to a correlation between the inflowing velocity and the inflowing temperature on seasonal time scales instead of being entirely due to baroclinic instability of the large-scale flow. The general properties of the circulation (temperature, horizontal circulation) are very similar to the standard case discussed here.

The relatively weak influence of eddies in balancing the surface loss differs from previous localized convection and periodic channel cases. The present configuration allows for two processes that were not permitted in these previous studies. The first is that the cooling takes place along open geostrophic contours which, for the present $f$-plane case, are topographic contours. They are referred to as "open" because all contours in the cooling region originate in the restoring region and thus may provide a source of heat. The second condition is that the model can develop a net temperature gradient along the direction of the flow. This is not allowed in periodic channel or closed basins with bowl topography configurations subject to cooling. Flow down (and up) the temperature gradient is found for closed topographic contours that extend through both heating and cooling regions (Nilsson et al., 2005). It is possible for the mean flow in a periodic channel to contribute to the heat flux through standing waves with a phase shift between temperature and velocity. However, with a flow along the temperature gradient, even weak mean flows can balance the surface heat loss. Time dependent eddies must be much stronger than the mean flow in order to achieve significant heat flux because the correlation between the eddy velocity and the eddy temperature anomaly is typically small.

\section{a. Closed topographic contours}

A calculation has also been carried out that has a region of closed topographic (geostrophic) contours in the northern part of the domain (Fig. 9). A dome of cold water lies over the region of closed contours, which is also encircled by a surface intensified cyclonic current. Consistent with previous studies of localized cooling, the surface heat flux in this region is balanced by lateral eddy fluxes and the mean advection is negligible. Eddy fluxes arise because there is a net cooling within the closed contours. The configuration of Nilsson et al. (2005) had both heating and cooling along closed contours, allowing for a mean barotropic flow to balance the local heating/cooling. The mean circulation outside of this region of closed topographic contours looks much like the standard case, with warm flow into the domain from the south and east and colder flow out of the domain in a narrower boundary current in the west. Mean advection is primarily responsible for balancing the surface cooling along these open topographic contours. The eddy fluxes are important of redistribution of heat within the basin by taking heat from the mean advection along open geostrophic contours and carrying it into the region of closed geostrophic contours. However, mean advection remains the dominant term for balancing the surface cooling when integrated over the whole basin. 


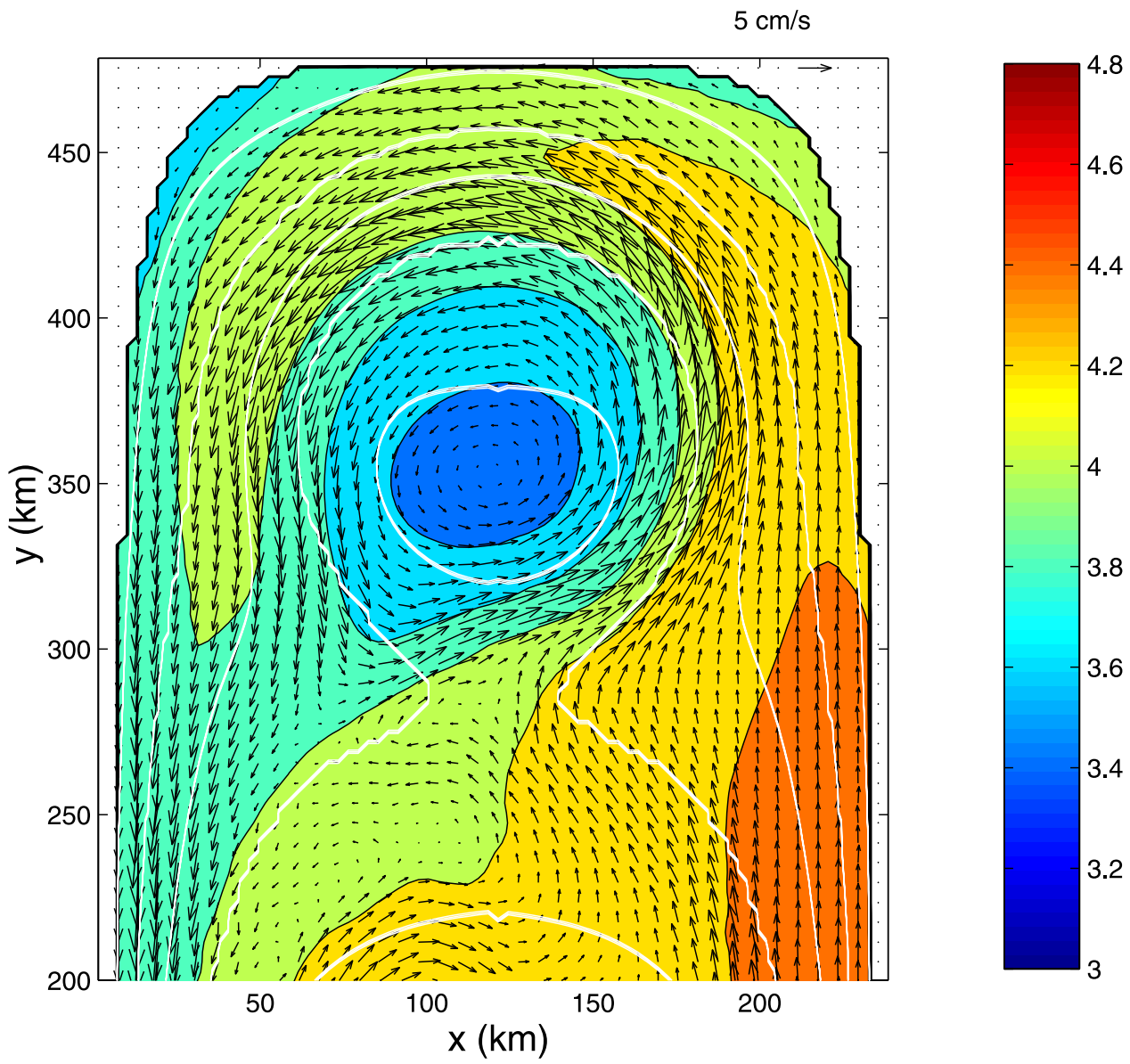

Figure 9. Mean temperature and velocity (every other grid point) at $25 \mathrm{~m}$ from the final 5 years of a model integration with a region of deep closed topographic contours (indicated by the white lines).

\section{b. Localized cooling}

The primitive equation model has been applied to the case with a localized patch of cooling over the sloping bottom shown in Figure 1. The forcing results in a patch of cool water that extends towards the south and west from the forcing region (Fig. 10). There is a weak, broad-scale barotropic flow of $O\left(1 \mathrm{~cm} \mathrm{~s}^{-1}\right)$ into the domain from the restoring region. This northward flow enters the cooling region, where it crosses the mean isotherms before turning towards the west and south in a strong, narrow current. This flow is analogous to the strong southward boundary current found in the uniform cooling case and eminates from the "downstream," in a characteristic sense, edge of the cooling region. The coldest waters are approximately $0.3^{\circ} \mathrm{C}$ cooler than the source waters.

The contributions from mean and eddy advection along depth contours are shown in Figure 11. The mean advection is everywhere warming and is strongest at depth. The 


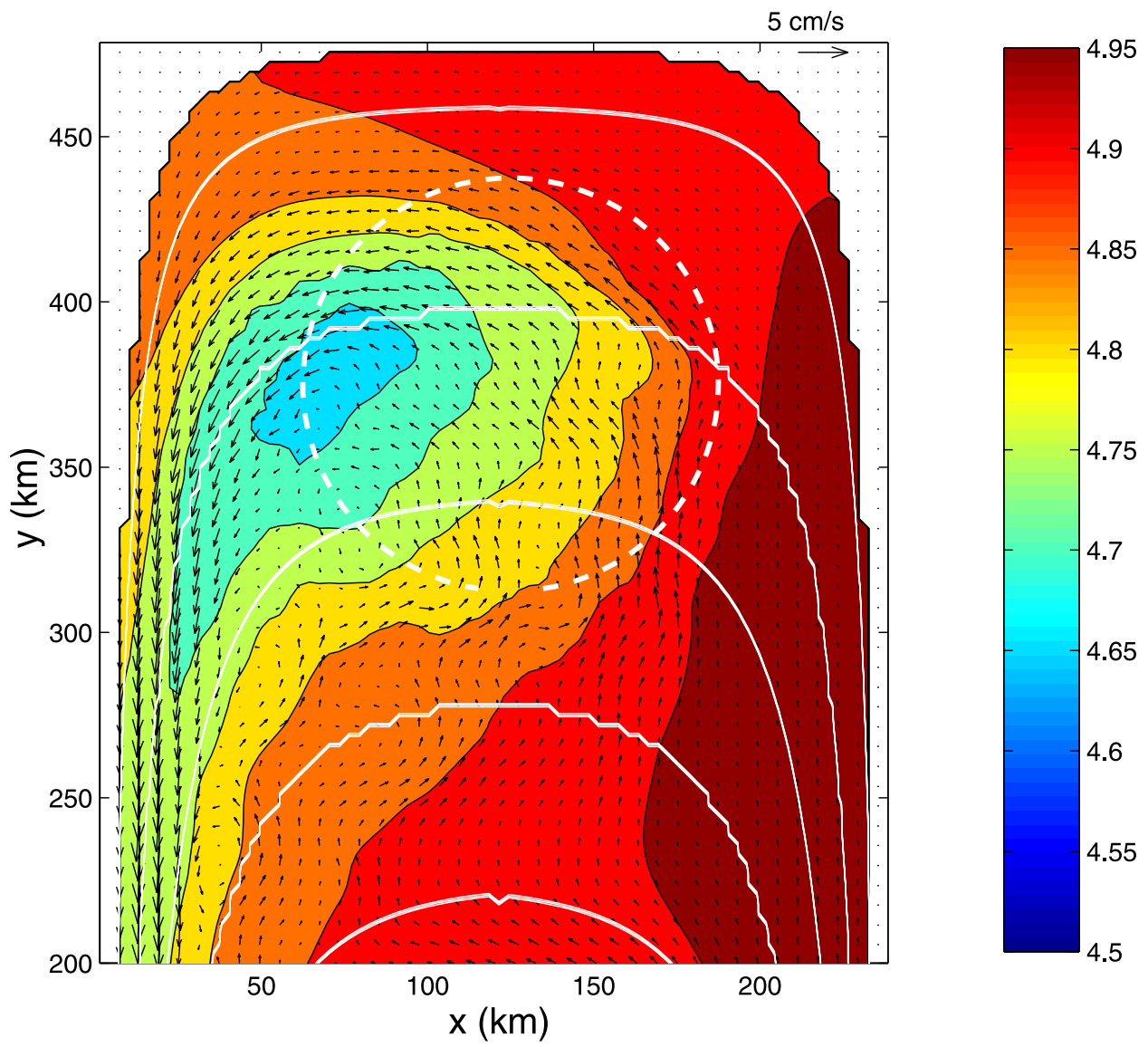

Figure 10. Mean upper level temperature and velocity (every other grid point) for a localized cooling within the white dashed circle. Bottom depth contours are shown in white.

eddies are warming the surface within the cooling region but are cooling the adjacent deep region. Mean advection is warming the deep region adjacent to the forcing region. Within the forcing region, both eddy and mean contributions are important, with the eddies slightly larger near the surface and the mean larger at depth. The depth-integrated contribution of the mean advection accounts for approximately twice as much warming as the depth-integrated eddy heat flux over the region of cooling. A similar calculation with the horizontal diffusion and viscosity increased by a factor of 8 suppresses baroclinic instability of the deep convection site. For this case, the eddy heat flux accounts for only $20 \%$ of the total. This suggests that some of the eddy heat flux is due to baroclinic instability of the rim current and some is due to a correlation between the large-scale horizontal advection and the large-scale temperature field on seasonal time scales.

The vertical integrals of the temperature tendency along depth contours reveal two distinct regions (Fig. 12). The eddies are warming the cooling region by advecting warm 

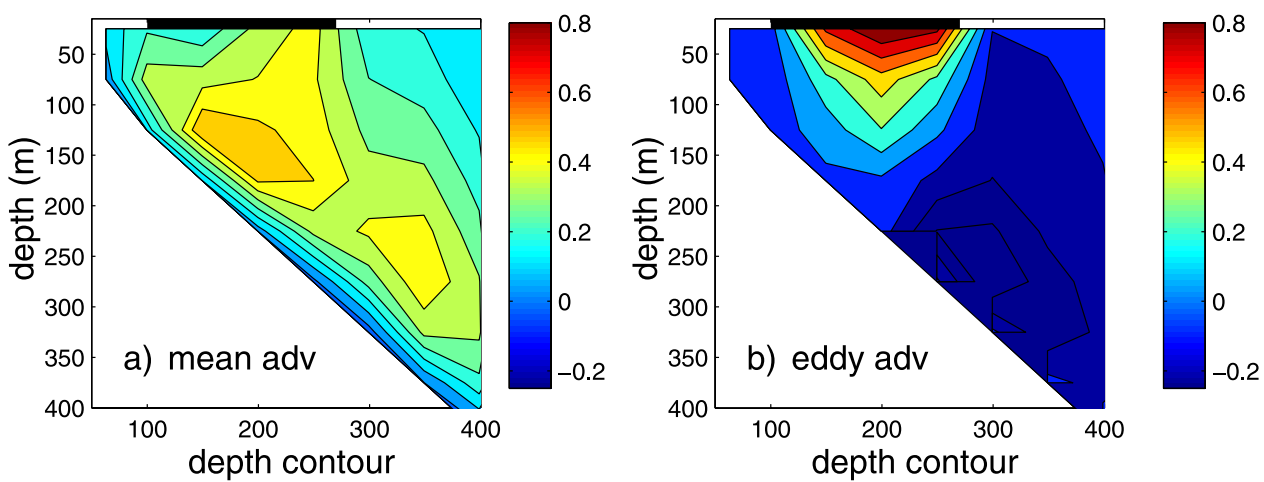

Figure 11. Contributions to the temperature tendency due to (a) mean advection and (b) eddy fluxes as a function of depth and depth contour for the case with a localized patch of cooling (units $\left.10^{-8}{ }^{\circ} \mathrm{C} \mathrm{s}^{-1}\right)$. The forcing region is indicated by the bold line near the surface.

water in from the deep region and exporting cool water to the deep region. Mean advection is warming the cooling region by an amount slightly greater than the eddies, but it is also warming the deep region to offset the cooling by the eddies. Eddies are important for restratification of the convection site, but require the mean advection along geostrophic contours to get the warm water from the restoring region to the region surrounding the cooling, similar to the case with closed topographic contours.

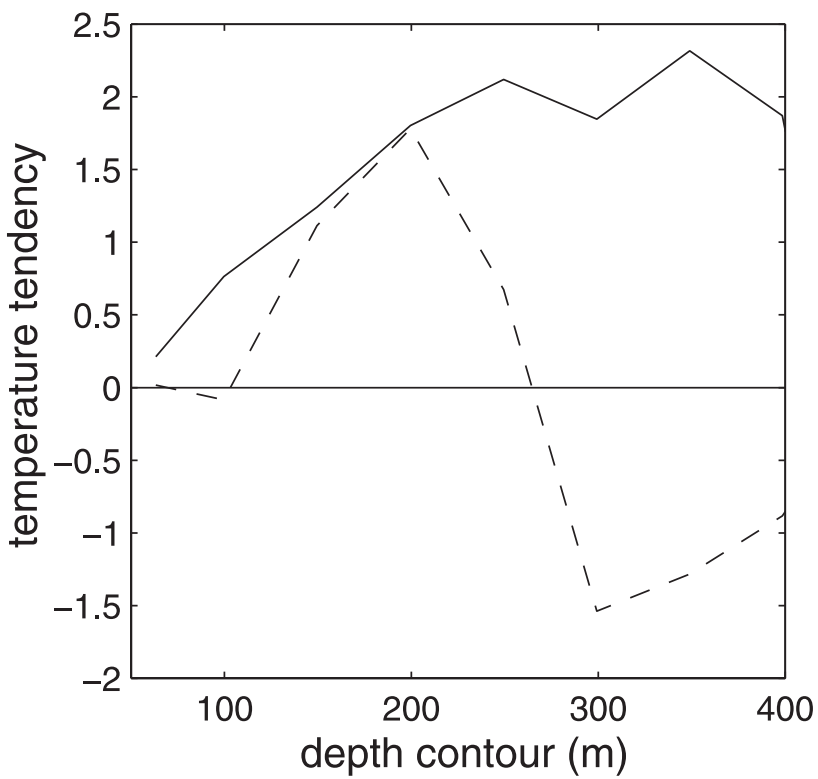

Figure 12. Vertical sum of the mean (solid) and eddy (dashed) heat flux divergences as a function of depth contour (units $10^{-8}{ }^{\circ} \mathrm{C} \mathrm{s}^{-1}$ ). 


\section{Simple models and parameter sensitivities}

The primitive equation model of the previous section provides the details of the temperature and velocity fields and includes many processes such as seasonal forcing, eddies, and frictional effects. However, the basic dynamics that govern the general behavior of the circulation and water mass transformation in the marginal sea are not readily apparent from these complex calculations. In this section, simple scaling ideas and an analytic two-layer planetary geostrophic model are compared to the primitive equation model and used to provide insights into what controls the water mass transformation and circulation in the marginal sea.

\section{a. Water mass transformation}

A heat budget for the marginal sea may be derived by balancing the lateral heat advection into and out of the marginal sea with the surface cooling within the marginal sea. A scaling is derived here that provides an estimate of the exchange rate and the temperature of the outflowing waters relative to the temperature of the inflowing waters. The change in temperature of the waters as they flow through the marginal sea is denoted as $\Delta T$. The heat budget within the marginal sea requires that

$$
M \Delta T=\frac{Q A}{\rho_{0} C_{p}},
$$

where $M$ is the mass transport flowing through the marginal sea, $Q$ is the surface heat flux (taken here to be uniform), $A$ is the surface area of the marginal sea, $\rho_{0}$ is the density of seawater, and $C_{p}$ is the specific heat of seawater. Note that contributions due to eddy heat fluxes between the marginal sea and the open ocean have been ignored. If it is assumed that the velocity of the inflowing and outflowing water is in geostrophic balance with the pressure gradient arising from the density anomaly, then the transport into the marginal sea scales with the change in temperature as

$$
M=\frac{\alpha g H^{2} \Delta T}{\rho_{0} f_{0}},
$$

where $H$ is a typical depth scale. Note that this relation is independent of the width of the basin (or current) but does assume that the convection reaches the bottom (or that the temperature anomaly $\Delta T$ reaches depth $H$ ).

Combining (1) and (2) provides an estimate for the change in temperature within the marginal sea

$$
\Delta T=\frac{1}{H}\left(\frac{Q A f_{0}}{\alpha g C_{p}}\right)^{1 / 2}
$$

The geostrophic velocity corresponding to this temperature change is

$$
V=\frac{1}{\rho_{0} L}\left(\frac{\alpha g Q A}{C_{p} f_{0}}\right)^{1 / 2}=\frac{g^{\prime} H}{L f_{0}} .
$$


Table 1. Model run parameters, units: $f_{0}\left(\mathrm{~s}^{-1}\right)$; mean heat flux $Q\left(\mathrm{~W} \mathrm{~m}^{-2}\right)$; topographic decay scale $l$ $(\mathrm{km})$; maximum depth $(\mathrm{m}) ; \Delta T\left({ }^{\circ} \mathrm{C}\right)$; transport through the marginal sea $M\left(10^{6} \mathrm{~m}^{3} \mathrm{~s}^{-1}\right)$ and symbols used in Figure 13.

\begin{tabular}{rrrrrrrl} 
RUN & \multicolumn{1}{c}{$f_{\mathrm{O}}$} & $Q$ & $l$ & Depth & $\Delta T$ & M & Symbol \\
1 & $1 \mathrm{e}-4$ & 20 & 25 & 500 & .46 & .58 & Square \\
2 & $3 \mathrm{e}-4$ & 20 & 25 & 500 & .71 & .39 & Asterisk \\
3 & $0.5 \mathrm{e}-4$ & 20 & 25 & 500 & .23 & 1.07 & Asterisk \\
4 & $0.25 \mathrm{e}-4$ & 20 & 25 & 500 & .11 & 1.79 & Asterisk \\
5 & $1 \mathrm{e}-4$ & 20 & 10 & 500 & .37 & .82 & Triangle \\
6 & $1 \mathrm{e}-4$ & 20 & 15 & 500 & .47 & .61 & Triangle \\
7 & $1 \mathrm{e}-4$ & 20 & 40 & 500 & .53 & .47 & Triangle \\
8 & $1 \mathrm{e}-4$ & 40 & 25 & 500 & .62 & .83 & Circle \\
9 & $1 \mathrm{e}-4$ & 10 & 25 & 500 & .32 & .47 & Circle \\
10 & $1 \mathrm{e}-4$ & 20 & 25 & 750 & .34 & .84 & Cross \\
11 & $1 \mathrm{e}-4$ & 20 & 25 & 250 & .80 & .33 & Cross \\
12 & $1 \mathrm{e}-4$ & 20 & 25 & 125 & 1.1 & .19 & Cross \\
13 & $0.5 \mathrm{e}-4$ & 20 & 15 & 750 & .13 & 1.87 & Diamond \\
14 & $2 \mathrm{e}-4$ & 40 & 25 & 250 & 1.69 & .30 & Diamond
\end{tabular}

The reduced gravity $g^{\prime}=\alpha \Delta T g / \rho_{0}$ and $L$ is the length scale of the cooling region. The velocity and temperature anomaly both increase with increasing heat loss and surface area of the marginal sea. The change in temperature in the marginal sea increases as the square root of the Coriolis parameter, while the velocity is inversely proportional to the square root of the Coriolis parameter. A small Coriolis parameter results in a larger geostrophic velocity to balance the density change, which in turn results in a smaller temperature change required to balance the heat loss. Shallower marginal seas result in larger changes in temperature because the heat loss is concentrated in a smaller volume of water. This scaling differs from previous ones by Visbeck et al. (1996), Chapman and Gawarkiewicz (1997), Marshall and Schott (1999), Pringle (2001), and Spall (2004), most notably because there is no contribution due to eddy fluxes and $\Delta T$ depends on $f_{0}$. A similar velocity scale is found by Nilsson et al. (2005) for the baroclinic circulation strength along closed topographic contours.

The velocity scale may also be interpreted as the baroclinic topographic Rossby wave speed. The variation in potential vorticity due to the sloping bottom may be written as $\beta=$ $s f_{0} / H$, where $s$ is the topographic slope. The long baroclinic Rossby wave phase speed is then $c=\beta L_{d}^{2}$, where $L_{d}=N H / f_{0}$ is the internal deformation radius and $N^{2}=g^{\prime} / H$ is the Brunt-Väisälä frequency. If it is assumed that $s$ scales as $H / L$, then the Rossby wave phase speed is identical to the above scaling (4) for $V$. This is also identical to the speed of an isolated dense lense of water on a sloping bottom derived formally by Nof (1983).

A series of primitive equation model calculations have been carried out in which the Coriolis parameter, the surface heat flux, the basic topography have been varied (see Table 1). The change in temperature within the marginal sea was calculated from zonal sections at $y=200 \mathrm{~km}$, as in Figure 5, by a flux weighted integral as 

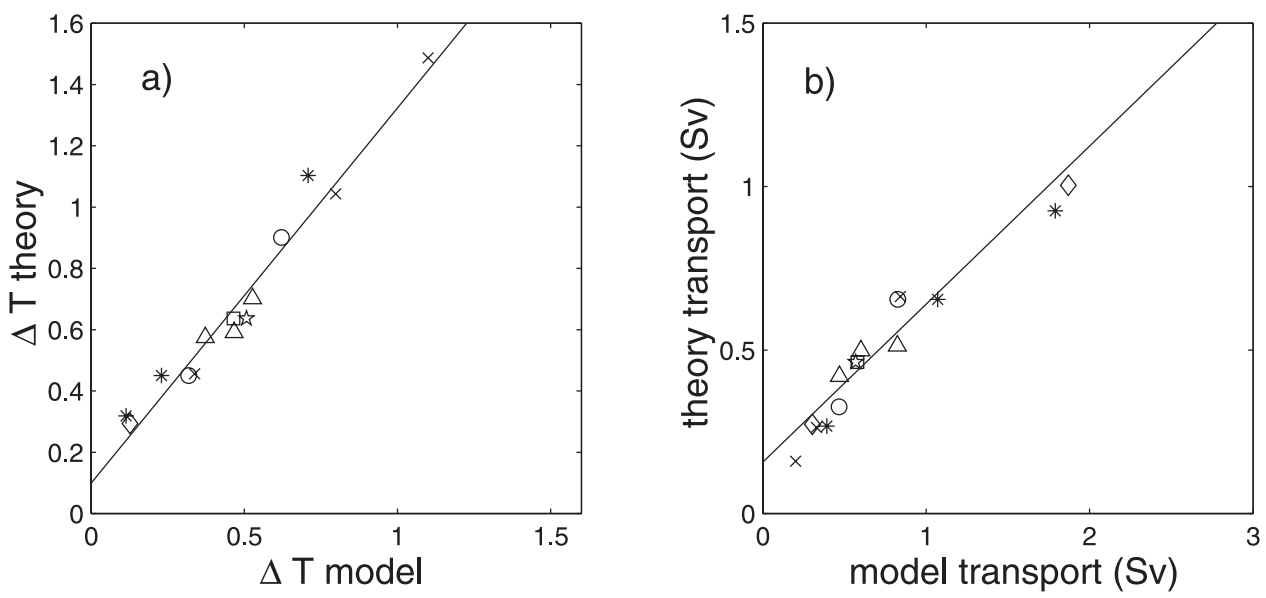

Figure 13. Comparison between the series of primitive equation model calculations (Table 1) and the theoretical estimates (3) and (2) for (a) the temperature anomaly of the outflowing waters and (b) exchange with the restoring region.

$$
\Delta T=\frac{\int_{0}^{L} \int_{0}^{D} v^{+} T d x d z}{\int_{0}^{L} \int_{0}^{D} v^{+} d x d z}-\frac{\int_{0}^{L} \int_{0}^{D} v^{-} T d x d z}{\int_{0}^{L} \int_{0}^{D} v^{-} d x d z}
$$

where $v^{+}$are all points with $v>0$ and $v^{-}$are points with $v<0, L$ is the basin width, and $D$ is the bottom depth. The theory is compared to the model results in Figure 13a. There is generally good agreement, with a correlation of 0.99 and a least square fit line of slope 1.22 .

The transport through the marginal sea has also been calculated from the model fields and compared to the scaling estimate (2), using (3). The theory compares well with the model, with a correlation of 0.96 and a least square fit line of slope 0.48 . The linear relationship verifies that the basic scaling and parameter dependencies are valid. The slope of less than 1 is not an indication of a failure in the method but instead indicates that one of the relationships (2) or (4) requires a scalar coefficient of $O(1)$. The transport varies between $0.20 \mathrm{~Sv}$ and $1.87 \mathrm{~Sv}$, even for cases in which the surface cooling is the same. The transport increases with decreasing Coriolis parameter and increasing basin depth.

\section{b. Two-layer circulation model}

A two-layer planetary geostrophic model is now used to illustrate the basic dynamics that control the horizontal and vertical circulation in the marginal sea. Buoyancy-forcing in this model is represented by a diapycnal velocity $w_{i}$, whose magnitude is imposed. A negative diapycnal velocity corresponds to converting warm water into cold water and thus represents cooling. Because the model allows for steep interface slopes, the diapycnal velocity is not purely vertical but may also include a contribution from horizontal advection through the sloping interface. The relative contributions due to horizontal and vertical advection are not specified but rather emerge as part of the solution. For simplicity 
in deriving the equations, it is assumed that the bottom topography is a function of $x$ only. The velocity in each layer may be written as

$$
\begin{aligned}
u_{2}=-\frac{g^{\prime} P_{y}}{f_{0}}-C_{d} v_{2} & v_{2}=\frac{g^{\prime} P_{x}}{f_{0}} \\
u_{1}=-\frac{g^{\prime}(P+h)_{y}}{f_{0}}-C_{d} v_{2} & v_{1}=\frac{g^{\prime}(P+h)_{x}}{f_{0}}
\end{aligned}
$$

where $P$ is the bottom pressure, $g^{\prime}=g\left(\rho_{2}-\rho_{1}\right) / \rho_{0}$ is the reduced gravity between the two layers, $h$ is the thickness of the upper layer, $C_{d}$ is a linear bottom drag coefficient, and the subscripts $x$ and $y$ refer to partial differentiation. It has been assumed that the layer 2 flow in the direction of the topography is much stronger than the flow across the topography, and so the bottom drag acts only on the along topography velocity $v_{2}$.

The continuity equation for the upper and lower layers may be written as

$$
\left(h u_{1}\right)_{x}+\left(h v_{1}\right)_{y}=w_{i} \quad\left[(D-h) u_{2}\right]_{x}+\left[(D-h) v_{2}\right]_{y}=-w_{i},
$$

where the depth of the ocean is $D$. Following the general approach of Luyten and Stommel (1986), the expressions for the horizontal velocities (6) may be substituted into the continuity equations and then summed to give a barotropic vorticity equation. The main differences with the model of Luyten and Stommel are that the present model is on an $f$-plane, has bottom topography, and has no wind forcing. The resulting vorticity equation is

$$
D_{x} P_{y}=-C_{d}\left(D P_{x}\right)_{x} .
$$

This relation balances the vertical velocity at the bottom due to a downslope flow (left-hand side) with the diffusion of relative vorticity in the bottom layer. The downslope flow is driven by the Ekman transport across depth contours. In the absence of frictional effects, the pressure is constant along lines of constant depth, $P_{y}=0$. This means that the deep flow is parallel to depth contours and its strength does not change in the downstream direction, although it can vary in the cross topography direction. This is consistent with the mean velocity at the bottom in the primitive equation model (Fig. 3), at least away from the region of strong baroclinic shear in the western part of the domain.

Neglecting frictional effects for now, the upper layer continuity equation, in terms of the velocity in layer 2 , simplifies to

$$
h_{y} v_{2}=w_{i}
$$

This shows that the diapycnal velocity is entirely due to horizontal advection across the sloping interface and that the vertical velocity in the interior, in the absence of friction, is zero. This is consistent with the mean vertical velocity in the primitive equation model. Note that this type of buoyancy-driven circulation could not be represented in a quasigeostrophic model since the diapycnal velocities would be required to be vertical. Such 
vertical motion would drive an erroneous basin-scale recirculation through constraints imposed by the vorticity equation.

This relation may be written in terms of the temperature of the upper layer as

$$
T_{y} v_{2}=\frac{Q}{\rho_{0} C_{p} D} .
$$

The solution for the upper layer temperature may be derived by integrating along the characteristics of the system, which in this case are simply lines of constant depth. The characteristic velocity is the along-topography velocity of the lower layer. The solution requires the specification of the transport per unit width $v_{2} D$ and the temperature of the upper layer on points where the characteristics flow into the domain.

A sample solution is given for a case with topography identical to that used for the primitive equation model. The inflow conditions are $T=5^{\circ} \mathrm{C}$ and $v_{2} D=V \bar{D}$, where $V=$ $\left(Q \alpha g A / C_{p} f_{0}\right)^{1 / 2} /\left(\rho_{0} L\right)=0.01 \mathrm{~m} \mathrm{~s}^{-1}$ is the scaling estimate for the inflowing velocity (4) and $\bar{D}$ is the average depth of the fluid on inflow. The transport has been (somewhat arbitrarily) assumed to be uniformly distributed over the inflowing characteristics. One could of course impose a more complex inflow but, lacking any other constraints, this seems the simplest choice that is broadly consistent with the circulation in the primitive equation model. For ease of numerical computation, it is assumed that $y$ is the direction along the topography and $x$ is the direction across the topography and the effect of curvature in the geostrophic contours is ignored.

The upper layer temperature is shown in Fig. 14a. For comparison, the mean upper level temperature in the central model calculation is shown with the same contouring and domain size in Figure 14b. The two-layer model reproduces well the basic characteristics of the full primitive equation model. The overall change in surface temperature is about 1 degree, with temperature decreasing cyclonically around the basin perimeter. Away from the lateral boundaries, the thermal wind between the upper and lower layer requires that the flow in the upper layer be towards the right, looking downstream, from the lower layer. This counterclockwise spiral of the velocity vector with depth is consistent with what was found in the primitive equation model. Such a geostrophic shear also results in a systematic transport in the upper layer from the deep ocean towards the basin perimeter such that outflowing waters are, on average, over shallower depth contours than the inflowing waters. In the standard primitive equation model calculation, the average depth of the inflowing waters is $324 \mathrm{~m}$ and the average depth of the outflowing waters is $237 \mathrm{~m}$.

The inviscid planetary geostrophic model can not satisfy the no-normal flow boundary condition along the boundary of the domain. The intersection of these temperature contours with the boundary requires the formation of a boundary layer that conserves mass and satisfies the boundary conditions. The development of a similar barotropic near boundary current as a result of downstream cooling, and a related asymmetry in the inflow and outflow widths, are discussed by Walin et al. (2004).

The two-layer planetary geostrophic model allows for a clear explanation of the westward intensification of the SST gradient. The characteristics enter the basin along the 

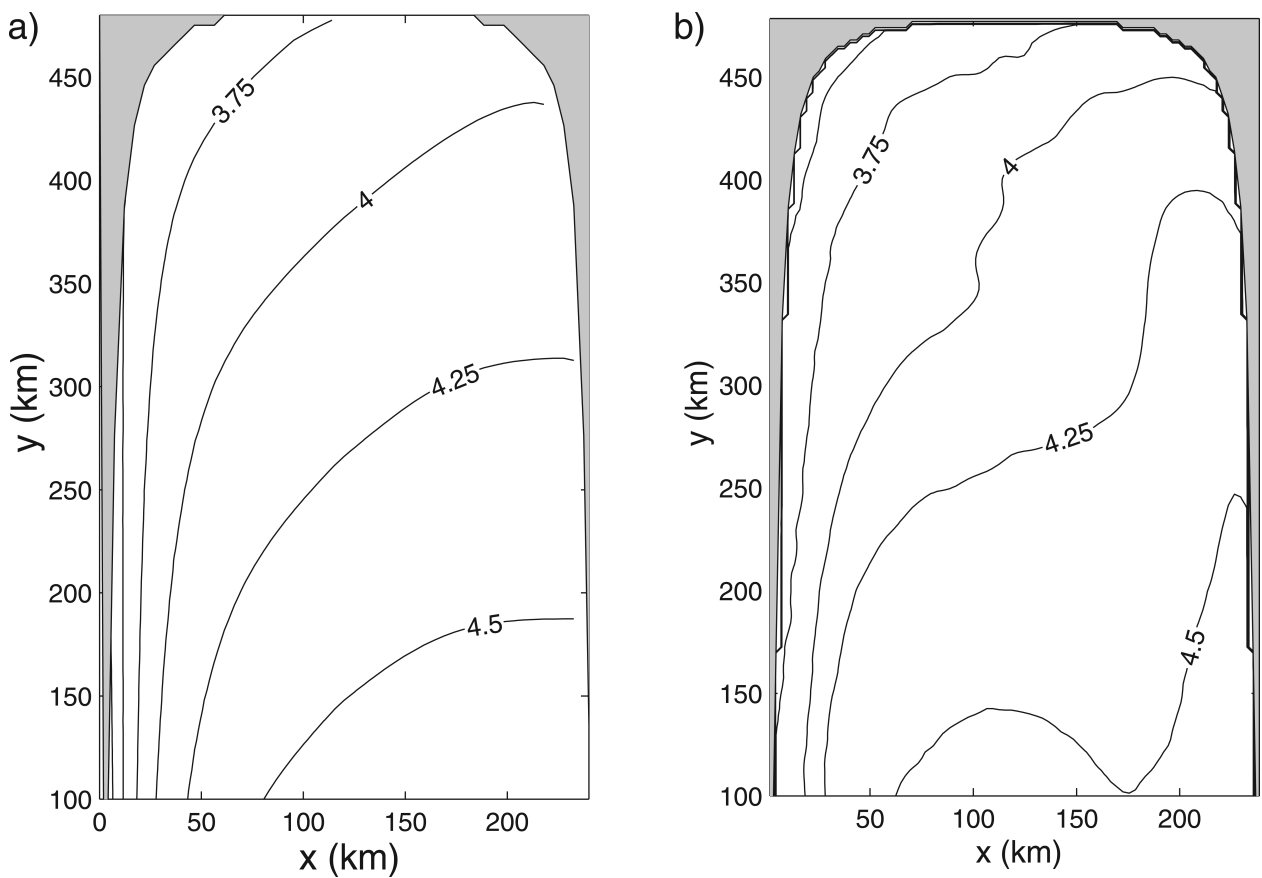

Figure 14. The upper layer temperature $\left({ }^{\circ} \mathrm{C}\right)$ from (a) the two-layer planetary geostrophic model, calculated by integrating (10) along geostrophic contours and (b) the primitive equation model.

southern boundary in the eastern half of the domain and exit the basin along the southern boundary in the western half of the domain. Note that it is the lower layer, or characteristic, velocity that controls the direction of information propagation and determines the upper layer temperature, not the upper layer velocity, which in some regions of the flow can be in opposite direction from the lower layer velocity. For a geostrophic flow, the baroclinic shear between the upper and lower layers can not contribute to the density advection, and so it is the deep velocity that controls the temperature field. The upper layer temperature decrease from the inflow temperature is due to the accumulation of heat loss along each characteristic trajectory. Those trajectories that enter the model domain in deep water exit the domain very quickly, but those that enter the domain in shallow water must travel farther around the outer perimeter of the model domain. This results in more heat loss for the shallow trajectories even though they ultimately exit the domain close to the deep trajectories, resulting in a large SST gradient. The arclength of each of the trajectories from their entry location, shown in Figure 15, clearly shows this effect.

The downslope flow and acceleration of the deep flow along the steep contours in the western part of the domain are not represented in this simple inviscid model, but may be explained by frictional effects. The presence of the boundary current that accumulates all of the upper layer transport directed normal to the boundary results in an increase in the along-topography transport $D P_{x}$ toward shallow water, so that $\left(D P_{x}\right)_{x}>0$. The 


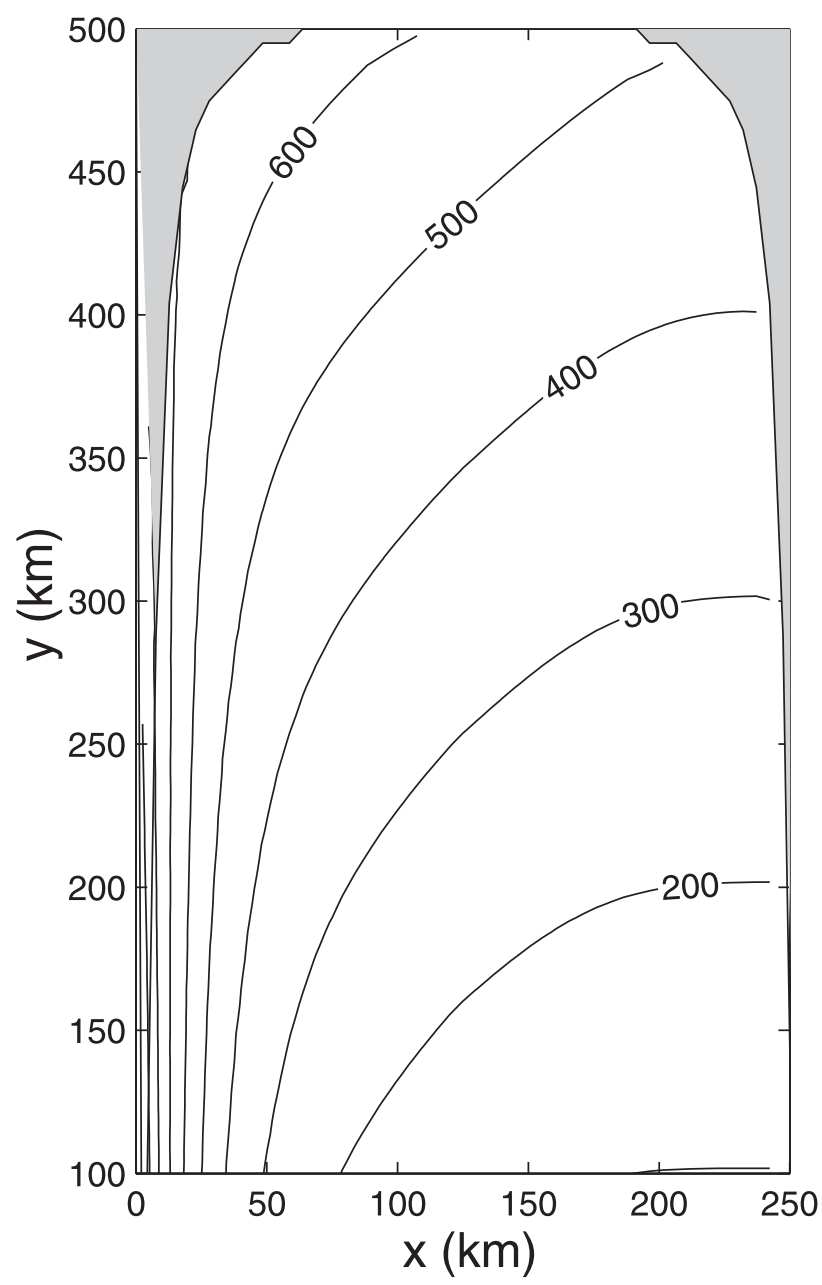

Figure 15. The distance (in $\mathrm{km}$ ) from the inflow boundary along geostrophic contours.

barotropic vorticity equation (8) requires then that the deep pressure increase along depth contours $\left(P_{y}>0\right)$, giving rise to a downslope flow and downward vertical velocity at the bottom. The increase in pressure along the topography will be largest near the boundary, where the gradients in transport and frictional effects are largest. The source of this downslope flow is the strong current flowing near the boundary. The increase in bottom pressure also enhances the deep transport and velocity above that that was specified upon inflow. This increases the characteristic velocity and thus will result in less cooling than is predicted by the inviscid model, as is found in the numerical model (although this is also in part due to eddy fluxes and slumping of the isopycnals). Although the frictional parameterization in this simple model is not the same as that in the primitive equation model, these features are consistent with the increase in deep transport and downslope flow found in just 
a)

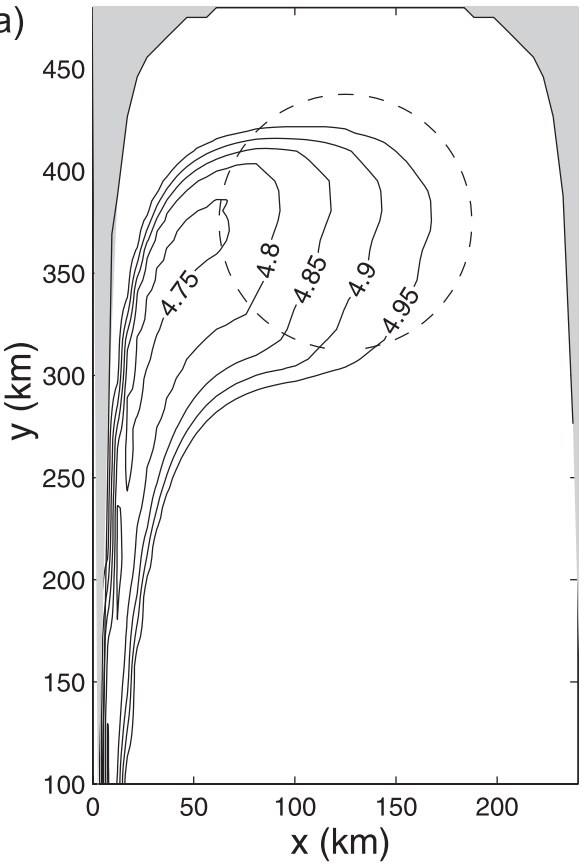

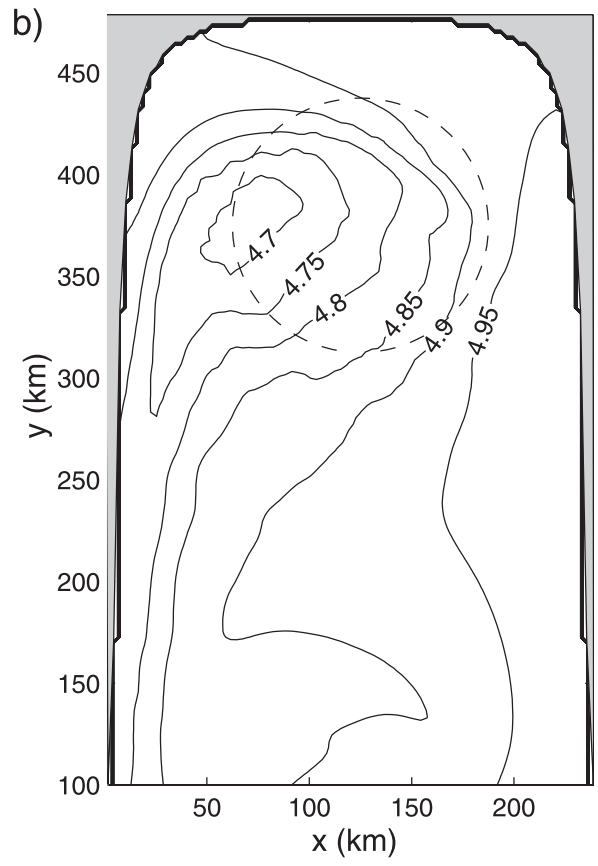

b)

Figure 16. Upper layer temperature $\left({ }^{\circ} \mathrm{C}\right)$ from (a) the two-layer planetary geostrophic model and (b) the primitive equation model. The region of cooling is indicated by the dashed line.

the region where the horizontal gradients in transport are large. The general behavior in the primitive equation model supports this interpretation in that increases in the viscosity result in an increase in the downwelling below the region of strong baroclinic shear.

The two-layer model has been applied to the problem forced by a circular region of cooling. The inflow velocity was taken from the scaling (2) with the $A$ given by the area, and inflow length scale $L$ by the diameter, of the cooling region. The pattern and magnitude of the sea surface temperature found in the primitive equation model are predicted well by the planetary geostrophic model (Fig. 16). The temperature patch in the primitive equation model appears slightly broader due to the influence of mesoscale eddies. The heat budget shows that the eddies, driven by baroclinic instability, act to restratify the cold plume extending from the forcing region. However, it is clear that the mean flow and topography exert the controlling influence on the density of the waters formed and the sea surface temperature.

\section{Summary and conclusions}

The buoyancy-driven circulation in shallow marginal seas has been explored using an idealized primitive equation numerical model and a two-layer planetary geostrophic model. The marginal sea was forced by surface cooling and connected to a source of warm water. The exchange rate between the marginal sea and the restoring region was not 
specified. Scaling theory was used to derive an estimate of the density anomaly of the waters formed within the marginal sea and the exchange rate with the open ocean. The theory assumes a balance between the mean flow and surface cooling, with no contribution due to mesoscale eddies. The theoretical estimate compares well with a series of numerical experiments in which the forcing and domain configuration parameters are varied. Heat budgets indicate that, for basin-scale forcing, the surface cooling is primarily balanced by mean horizontal advection governed by the deep flow along topographic contours. Although mesoscale eddies develop at the end of the cooling period, they mainly provide a local restratification of the water column and contribute little depth integrated lateral heat transport. This is in contrast to many previous studies of isolated deep convection. For cases of a localized cooling patch, eddies are found to be more important, but still less so than mean advection along topography.

The general pattern and amplitude of the sea surface temperature for both the basin-scale and localized patch of cooling are well predicted by a simple two-layer planetary geostrophic model. This agreement supports the conclusion that the basic dynamics are a balance between horizontal advection along geostrophic contours at the baroclinic Rossby wave phase speed and surface cooling. The strong frontal regions that develop along the western boundary are a result of the convergence of geostrophic contours that have experienced different amounts of cooling along their paths through the basin.

Perhaps the most important result of this study is the finding that, even though eddies are generated in regions of deep convection, they do not play a central role in balancing the local heat loss. This differs from previous studies in which eddies were found to be crucial for balancing surface cooling. The weaker role of eddies in the present study is a result of removing a degree of symmetry in the along-flow direction. Heat transport by eddies has primarily been found to be important for cases in which the mean streamlines are closed (e.g. Visbeck et al., 1996; Pringle, 2001), either through localized cooling or enforcing periodicity in the along flow direction. The present configuration allows for a change in the density in the along-flow direction, thus allowing for the mean flow to cross mean temperature contours over the extent of the basin.

Acknowledgments. This work was supported by the Office of Naval Research under Grant N00014-03-1-0338 and by the National Science Foundation under Grant OCE-0240978. Göran Broström and an anonymous reviewer provided many useful comments and suggestions. This is Woods Hole Oceanographic Institution contribution number 11315.

\section{REFERENCES}

Chapman, D. C. and G. Gawarkiewicz. 1997. Shallow convection and buoyancy equilibration in an idealized coastal polynya. J Phys. Oceanogr., 27, 556-566.

Condie, S. A. and P. B. Rhines. 1994. Topographic Hadley cells. J Fluid Mech., 280, 349-368.

Luyten, J. and H. Stommel. 1986. Gyres driven by combined wind and buoyancy flux. J. Phys. Oceanogr., 16, 1551-1560.

Marshall, J. and F. Schott. 1999. Open-ocean convection: observations, theory, and models. Rev. Geophys. 37, 1-64.

Marshall, J., C. Hill, L. Perelman and A. Adcroft. 1997. Hydrostatic, quasi-hydrostatic, and nonhydrostatic ocean modeling. J. Geophys. Res., 102, 5733-5752. 
Nilsson, J. G. Walin and G. Broström. 2005. Thermohaline circulation induced by bottom friction in sloping-boundary basins. J. Mar. Res., 63, 705-728.

Nof, D. 1983. The translation of isolated cold eddies on a sloping bottom. Deep-Sea Res., 30, $171-182$.

Pringle, J. M. 2001. Cross-shelf eddy heat transport in a wind-free coastal ocean undergoing winter time cooling. J. Geophys. Res., 106, 2589-2604.

Rhines, P. B. 1998. Circulation, convection and mixing in rotating, stratified basins with sloping topography, in Physical Processes in Lakes and Oceans, J. Imberger, ed., AGU Coastal and Estuarine Series, 54, 435-451.

Spall, M. A. 2004. Boundary currents and water mass transformation in marginal seas. J. Phys. Oceanogr., 34, 1197-1213.

Straub, D. N. and P. B. Rhines. 1990. Effects of large-scale topography on abyssal circulation. J. Mar. Res., 48, 223-253.

Visbeck, M., J. Marshall and H. Jones. 1996. Dynamics of isolated convective regions in the ocean. J. Phys. Oceanogr., 26, 1721-1734.

Walin, G., G. Broström, J. Nilsson and O. Dahl. 2004. Baroclinic boundary currents with downstream decreasing buoyancy; a study of an idealized Nordic Seas system. J. Mar. Res., 62, $\underline{517-543 .}$

Zavatarelli, M., N. Pinardi, V. H. Kourafalou and A. Maggiore. 2002. Diagnostic and prognostic model studies of the Adriatic Sea general circulation: Seasonal variability. J. Geophys. Res., 107(C1), 3004, doi:10.1029/2000JC000210.

Received: 13 October, 2004; revised: 24 February, 2005. 